\title{
openheart Mild cognitive impairment impacts health outcomes of patients with atrial fibrillation undergoing a disease management intervention
}

\author{
Jocasta Ball, ${ }^{1,2}$ Maja-Lisa Løchen, ${ }^{1,3,4}$ Melinda J Carrington, ${ }^{1,2,4}$ Joshua F Wiley, ${ }^{5}$ \\ Simon Stewart ${ }^{4}$
}

\begin{abstract}
- Additional material is published online only. To view please visit the journal online (http://dx.doi.org/10.1136/ openhrt-2017-000755).
\end{abstract}

To cite: Ball J, Løchen M-L, Carrington MJ, et al. Mild cognitive impairment impacts health outcomes of patients with atrial fibrillation undergoing a disease management intervention. Open Heart 2018;5:e000755. doi:10.1136/ openhrt-2017-000755

Received 1 December 2017 Revised 12 December 2017 Accepted 29 December 2017

Check for updates

${ }^{1}$ Pre-Clinical Disease and Prevention, Baker Heart and Diabetes Institute, Melbourne, Victoria, Australia

${ }^{2}$ School of Public Health and Preventive Medicine, Monash University, Melbourne, Victoria Australia

${ }^{3}$ Department of Community Medicine, UiT The Arctic University of Norway, Tromsø, Norway

${ }^{4}$ Mary MacKillop Institute for Health Research, Australian Catholic University, Melbourne, Victoria, Australia

${ }^{5}$ School of Psychological Sciences, Monash University, Clayton, Victoria, Australia

Correspondence to

Dr Jocasta Ball; Jocasta.Ball@ baker.edu.au

\section{ABSTRACT}

Objective Mild cognitive impairment $(\mathrm{MCl})$ is prevalent in atrial fibrillation (AF) and has the potential to contribute to poor outcomes. We investigated the influence of $\mathrm{MCl}$ on survival and rehospitalisation in patients with chronic forms of AF undergoing a home-based, AF-specific disease management intervention (home-based intervention (HBI)) or standard management (SM).

Methods The Montreal Cognitive Assessment tool was administered at baseline (a score of $<26 / 30$ indicated MCI) in patients with $\mathrm{AF}$ randomised to $\mathrm{HBI}$ versus SM. Post hoc analyses of mortality and rehospitalisations during a minimum 24-month follow-up were conducted in the overall cohort and in each study group separately. Results of 260 patients (mean age $72 \pm 11,47 \%$ female), $65 \%$ demonstrated $\mathrm{MCl}$ on screening (34\% in SM; $31 \%$ in $\mathrm{HBI})$. Overall, the number of days spent alive and outof-hospital during follow-up ( $\mathrm{P}=0.012$ ) and all-cause rehospitalisation were influenced by $\mathrm{MCl}$ during follow-up (OR 3.16 (95\% $\mathrm{Cl} 1.46$ to 6.84$)$ ) but $\mathrm{MCl}$ did not influence any outcomes in the SM group. However, survival was negatively influenced by $\mathrm{MCl}$ in the $\mathrm{HBI}$ group $(\mathrm{P}=0.036)$; those with $\mathrm{MCl}$ in this group were 5.6 times more likely to die during follow-up (OR 5.57 (95\% Cl 1.10 to 28.1)). Those with MCl in the HBI group also spent less days alive and out-of-hospital than those with no $\mathrm{MCl}(\mathrm{P}=0.022)$. $\mathrm{MCl}$ was also identified as a significant independent correlate of shortest duration of event-free survival (OR $3.48(95 \% \mathrm{Cl} 1.06$ to 11.4$))$, allcause rehospitalisation (0R $3.30(95 \% \mathrm{Cl} 1.25$ to 8.69$)$ ) and cardiovascular disease (CVD)-related rehospitalisation (OR $2.35(95 \% \mathrm{Cl} 1.12$ to 4.91$))$ in this group.

Conclusions The effectiveness of home-based, disease management for patients with chronic forms of AF is negatively affected by comorbid $\mathrm{MCl}$. The benefit of adjunctive support for patients with MCl on CVD-related health outcomes requires further investigation.

\section{INTRODUCTION}

Atrial fibrillation (AF) is the most common cardiac rhythm disturbance observed in clinical practice. $\mathrm{AF}$ is prevalent in $2 \%-3 \%$ of the general population, and more than $70 \%$ of individuals with $\mathrm{AF}$ are aged 65 years and older. ${ }^{1}$ Prevalence of AF is rising in parallel to population ageing, improved survival from

\section{Key questions}

What is already known about this subject?

- Mild cognitive impairment (MCI) is a common comorbidity in atrial fibrillation (AF) and influences a patient's ability to self-care and perform simple daily tasks. Cognitive impairment is becoming increasingly recognised as exerting a powerful and negative impact on the risk profile, management and prognosis of patients with AF.

What does this study add?

- Patient outcomes of a cohort of patients with AF were affected by MCl. The presence of $\mathrm{MCl}$ in patients with $\mathrm{AF}$ undergoing standard postdischarge management did not influence survival or rehospitalisation. However, the benefit of a postdischarge, AF-specific disease management intervention was negatively influenced by the presence of $\mathrm{MCl}$. The risk of death for AF patients with comorbid $\mathrm{MCl}$ in the intervention group was increased 5.6-fold, and $\mathrm{MCl}$ was the only independent predictor of rehospitalisation during minimum 24-month follow-up, increasing the risk by over threefold. Patients without $\mathrm{MCl}$ in the intervention group had significantly less unplanned, all-cause and cardiovascular-specific hospitalisations during follow-up compared with those with $\mathrm{MCl}$.

How might this impact on clinical practice?

- Successful patient care can be influenced by the presence of $\mathrm{MCl}$. Therefore, all older patients with AF should be screened for $\mathrm{MCI}$ to allow care to be individualised and tailored accordingly. Adjunctive support for those with $\mathrm{MCl}$ may go somewhat towards optimising the health outcomes of these patients.

other clinical conditions that increase the likelihood of AF development and little progress in reducing major significant risk factors such as obesity. ${ }^{1}$ Multiple complications are the direct result of $\mathrm{AF}$, including thromboembolic events (notably stroke), heart failure, reduced quality-of-life and death. ${ }^{23}$ 
$\mathrm{AF}$ is associated with the most common forms of thromboembolism (stroke risk is increased fivefold in patients with $\mathrm{AF}$ ) and responsible for subtle brain changes due to the occurrence of silent cerebral infarcts, microemboli, microbleeds and cerebral hypoperfusion. ${ }^{4-8}$ The result is gradual cognitive dysfunction beginning with mild cognitive impairment (MCI), which markedly influences the ability of patients to self-care and perform simple daily tasks such as taking prescribed medications. ${ }^{9} \mathrm{AF}$ is independently associated with cognitive decline, and patients with $\mathrm{AF}$ are at twofold higher risk of cognitive impairment than age-matched patients without AF. ${ }^{10}{ }^{11}$ Cognitive impairment is becoming increasingly recognised as exerting a powerful and negative impact on the risk profile, management and prognosis of patients with $\mathrm{AF}^{12}$ Higher rates of morbidity, symptom severity, healthcare utilisation and mortality resulting from the negative impact of cognitive impairment have been observed in older individuals (some with heart failure and other cardiovascular conditions $)^{13-16}$ and the same is likely to be observed in AF populations. AF patients with cognitive impairment have poorer anticoagulation adherence, more frequent cardiovascular events and demonstrate greater functional impairment compared with those without cognitive impairment. ${ }^{12}$ Additionally, patients with MCI are vulnerable to mood disorders including depression ${ }^{17}$ with psychosocial and cognitive comorbidities clustering frequently in patients with $\mathrm{AF}^{14}$ The resulting psychosocial and cognitive multimorbidity exerts a powerful and negative effect on AF-specific quality of life. $^{14}$

With the continued evolution of individualised care to optimise AF management, ${ }^{18}$ it is imperative to understand the influence of cognitive impairment on the effectiveness of care for patients with AF. Therefore, we aimed to investigate the influence of MCI on the outcomes (namely mortality, days alive and out-of-hospital and rehospitalisations) of patients hospitalised as a result of AF. Furthermore, we aimed to examine the impact of MCI on the outcomes of patients participating in an AF-specific disease management programme compared with those undergoing standard postdischarge management.

\section{METHODS \\ Study setting}

Details of the Standard versus Atrial Fibrillation spEcific managemenT strategY (SAFETY) Trial protocol ${ }^{19}$ and primary composite endpoint outcomes of death or unplanned rehospitalisation due to any cause ${ }^{20}$ have been reported previously. Briefly, 335 inpatients $(52 \%$ male) from three tertiary referral hospitals in Australia with a diagnosis of chronic forms of AF (recurrent paroxysmal, persistent or permanent $\mathrm{AF}$ ) were randomly assigned into the study. Participants were randomised (via a computer-generated protocol and using block groups) to either standard management (SM; n=167) or an AF-specific home-based management intervention
(HBI; n=168) aimed at reducing morbidity and mortality following hospital discharge. Nurse-led clinic visits were conducted at 12 and 24 months for follow-up assessment of surviving patients, with comprehensive details of all deaths and rehospitalisations collected.

The SAFETY Trial was prospectively registered with the Australian New Zealand Clinical Trials Registry (ANZCTRN: 12610000221055) and was Consolidated Standards of Reporting Trials compliant with outcomes reported appropriately for a pragmatic trial comparing the efficacy of two non-pharmacological health interventions. ${ }^{21}$ Written informed consent was obtained from each study participant prior to any study procedure being conducted. The study protocol conformed to the ethical guidelines of the Declaration of Helsinki.

\section{Participants}

Using a systematic screening programme to identify eligible participants, inpatients were approached for recruitment if they were aged 45 years or older, had a documented diagnosis of a chronic form of AF (persistent or permanent $\mathrm{AF}$ ), would be living independently in the community or their own home postdischarge and were able and willing to provide written informed consent to participate. Those who had a primary diagnosis of valvular heart disease, had a transient form of AF, were scheduled for catheter ablation and/or had concurrent heart failure (confirmed on echocardiography (including screening for evidence of diastolic heart failure) and defined as displaying New York Heart Association class III-IV symptoms with a documented left ventricular ejection fraction of less than $45 \%$ ) were excluded from participation. Also excluded from participation were those who had a documented diagnosis of cognitive impairment, delirium or dementia and those who were not proficient in understanding or conversing in English (ie, those unable to provide informed consent).

\section{Baseline cohort profiling}

Comprehensive baseline profiling was conducted in all participants during index hospitalisation. Data were collected on sociodemographic status, past medical history (including AF-specific details), inhospital and planned postdischarge management, prescribed therapeutics, thromboembolic risk (calculated by the $\mathrm{CHA}_{2} \mathrm{DS}_{2}$-VASc score ${ }^{22}$ ), comorbid conditions including calculation of an age-adjusted Charlson Comorbidity Index score ${ }^{23}$ and cognitive status (determined by the Montreal Cognitive Assessment (MoCA) tool $^{24}$ ). The MoCA is a $10 \mathrm{~min}, 30$-item screening tool for MCI. A cut-off score of 26 (range 0-30) has been shown to have high sensitivity $(0.90)$ and specificity $(0.87)$ for detecting MCI. ${ }^{24}$ To account for differences in education attained, one point is added to the total score for those with $\leq 12$ years of formal education (education level-corrected scores of below 26 are considered as being cognitively 'impaired'). ${ }^{24}$ The MoCA was administered to $n=260$ of the total cohort of 335 patients. Patients who did not 
undertake the MoCA were those who were physically unable to do so (eg, due to vision disturbance) and those from non-English speaking backgrounds who may have been proficient in English but for whom the MoCA has not been validated.

\section{Study intervention}

The study intervention involved proactive patient management to optimise gold standard pharmacological and non-pharmacological management according to guidelines. Structured postdischarge care consisted of an initial home visit conducted by an intervention nurse at 7-14 days postdischarge and a combination of telephone follow-up and repeat home visits. The initial home visit, which involved a comprehensive assessment of each patient, was performed including evaluation of medication adherence, self-care, disease knowledge and clinical status (blood pressure, international normalised ratio where appropriate, current symptoms and heart rate and rhythm (12-lead ECG)). A postvisit needs assessment of each participant was conducted including risk stratification based on visit findings and determination of level of intervention required to maintain (or achieve) clinical stability during follow-up. Clinic reviews were conducted at 12 and 24 months postdischarge. Components of the individualised management were specifically focused on $\mathrm{AF}$ and included advanced clinical assessment (including the use of Holter monitoring), a review of social circumstances, education, review of medications and treatment plan according to gold standards, identification of barriers to self-care and communication with or referral to other healthcare providers and community services.

\section{Statistical analyses}

Descriptive data are presented as mean (with $\mathrm{SD}$ ) or median (with IQR) for continuous variables or as a proportion (\%) for categorical variables. Between-group comparisons were conducted with Student's t-test, $\chi^{2}$ test or Mann-Whitney U test where appropriate.

We performed Kaplan-Meier survival analyses in the overall cohort and in the SM and HBI groups separately to assess survival from all-cause mortality and time to the primary endpoint of mortality and unplanned readmission (measured as days alive and out-of-hospital) in participants classified as having MCI (MoCA scores <26/30) versus those of normal cognition (MoCA scores $\geq 26 / 30$ ). Group comparisons were performed with a log-rank test. Mean survival time (with 95\% CIs) was calculated for each respective group. As the number of potential covariates was large relative to the sample size, only covariates that were empirically related to outcomes were included. Covariates were identified using multiple logistic regression with backwards, stepwise selection for: all-cause mortality, inclusion in the lower quartile of days alive and out-of-hospital ( $\leq 763$ days), all-cause hospital admission and hospital admission due to cardiovascular disease (CVD). All multiple logistic regression models included sociodemographic (age and gender), lifestyle (current smoking), medical history/status (MCI, hypertension, cerebrovascular disease and type 2 diabetes) and treatment factors (rate/rhythm control, beta blocker, calcium channel blocker, ACE inhibitor, angiotensin II receptor blocker, digoxin and warfarin prescription), and all models were stratified by group of randomisation (SM vs HBI). MCI was initially entered into all statistical models as a binary variable ('Yes' vs 'No'). To test the interaction of randomisation group with MCI status, we replaced MCI in the overall regression models with a four-group variable (noMCI-HBI, MCI-HBI, noMCI-SM and MCI-SM). All data were analysed using SPSS V.22.0. A probability value of $\mathrm{P}<0.05$ (two sided) was considered statistically significant.

\section{RESULTS \\ Baseline profile}

Baseline characteristics of SAFETY participants stratified by randomisation group and MCI are presented in table 1 . Of the total cohort, $65 \%$ demonstrated MCI on screening $(\mathrm{n}=89(34 \%)$ in SM and $\mathrm{n}=80(31 \%)$ in HBI). Overall, this cohort included older patients (mean age $72 \pm 11$ years) at high thromboembolic and clinical risk (mean $\mathrm{CHA}_{2} \mathrm{DS}_{2}$-VASc score 3.6 \pm 1.8 ) and suffering from multimorbidity (mean Charlson Comorbidity Index 5.0 2.5 ). Within the SM group, those with MCI were significantly older (mean age 74 years vs 68 years, respectively), at higher thromboembolic risk (mean $\mathrm{CHA}_{2} \mathrm{DS}_{2}$-VASc score 4.0 vs 2.8, respectively) and suffering from significantly more comorbidity (mean Charlson Comorbidity Index score 5.6 vs 4.3 , respectively) than those without MCI. The same patterns were identified within the HBI group. Although, in addition, significantly less participants with MCI obtained tertiary-level education (24\% vs $44 \%$, respectively) and significantly more suffered from vascular disease and were prescribed digoxin therapy compared with those without MCI ( $10 \%$ vs $0 \%$ and $44 \%$ vs $23 \%$, respectively).

\section{Survival}

All-cause mortality

Figure 1 shows the unadjusted survival curves of participants with and without MCI in the overall cohort (figure 1A) and in the SM (figure 1B) and HBI (figure 1C) groups separately. Over a mean follow-up of $879 \pm 299$ days, 40 individuals died overall $(15.4 \%)$ including 31/169 participants (18.3\%) with MCI and 9/91 (9.9\%) without MCI. Mean survival time in these groups was 1209 days (95\% CI 1149 to 1269 days) and 1255 days (95\% CI 1187 to 1324) days, respectively. Within the SM group, over a mean follow-up of $874 \pm 289$ days, 24 individuals died (18.2\%) including $17 / 89$ participants $(19.1 \%)$ with MCI and $7 / 43(16.3 \%)$ without MCI. Mean survival time in these groups was 1206 days (95\% CI 1129 to 1284 days) and 1187 days (95\% CI 1068 to 1306 days), respectively. Within the HBI group, over a mean follow-up of $884 \pm 310$ days, 16 individuals died (12.5\%) including 14/80 


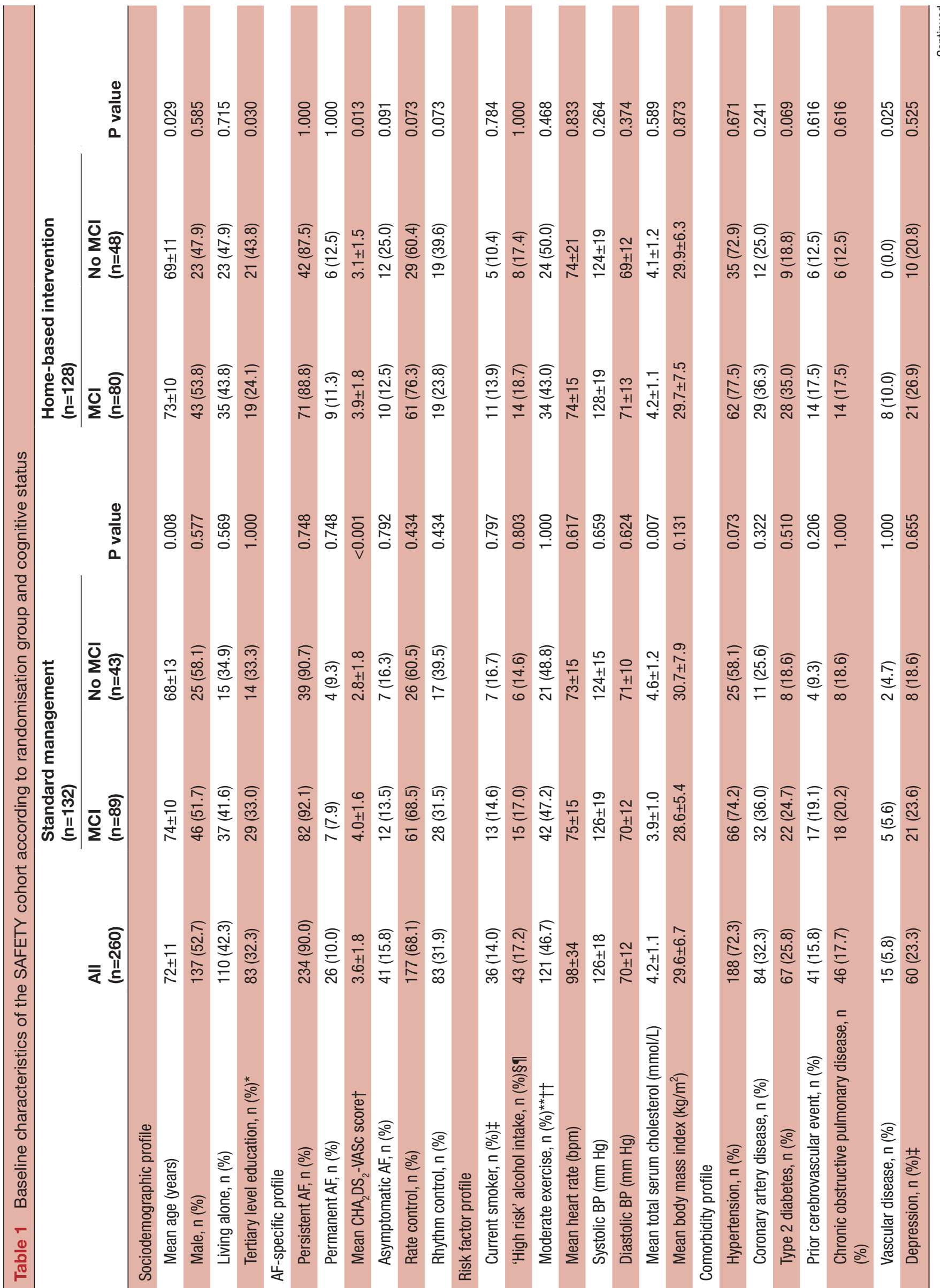




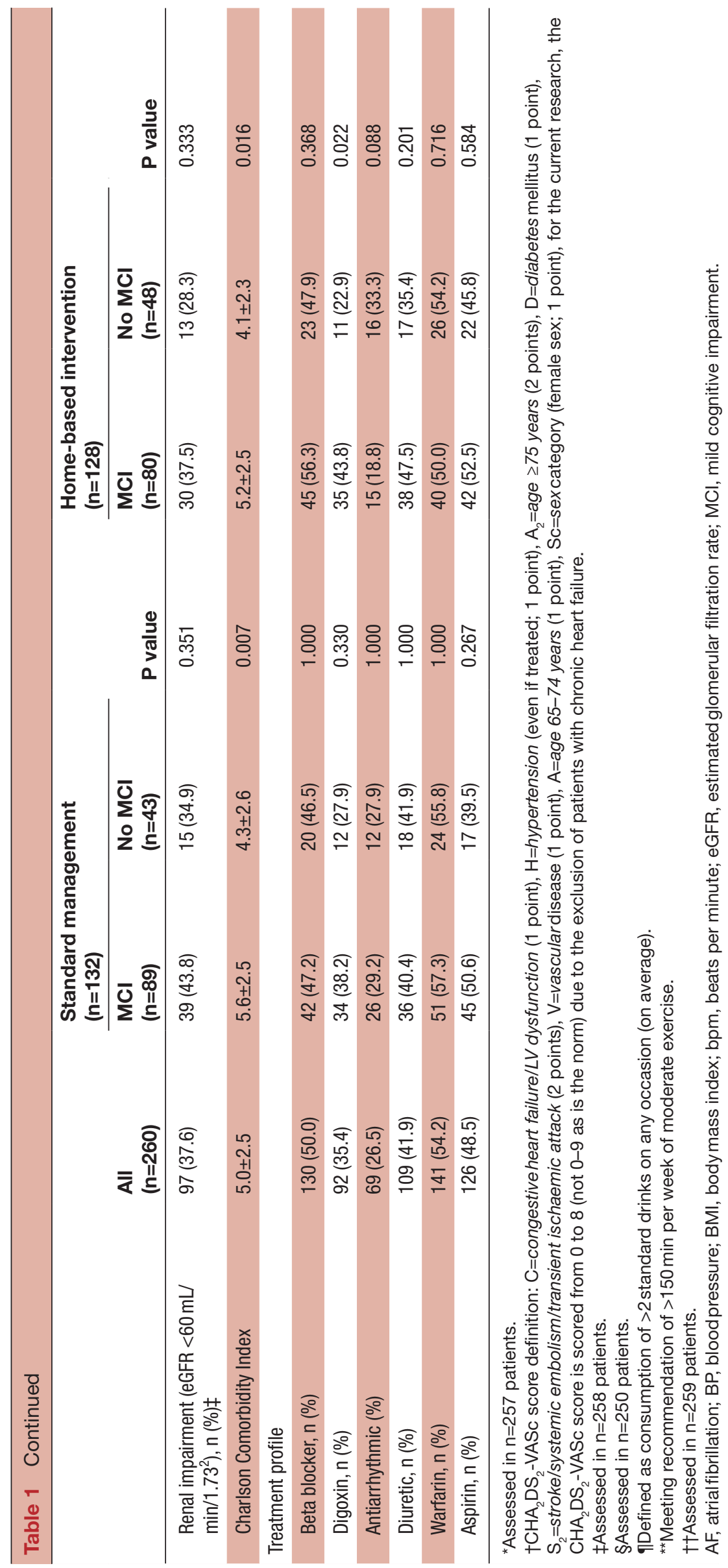



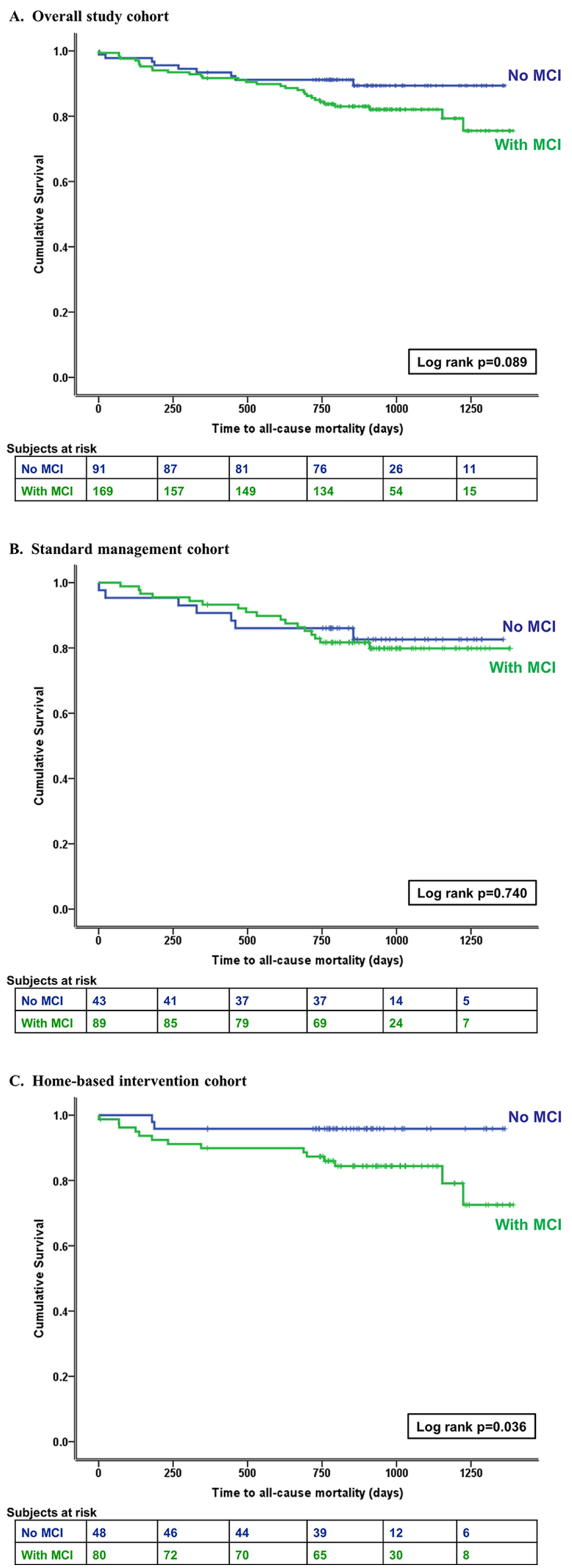

Figure 1 Unadjusted survival from all-cause mortality of participants with and without $\mathrm{MCl}$ in (A) overall cohort, (B) standard management cohort and (C) home-based intervention cohort. $\mathrm{MCl}$, mild cognitive impairment. participants (17.5\%) with MCI and 2/48 (4.2\%) without MCI. Mean survival time in these groups was 1206 days (95\% CI 1116 to 1297 ) days and 1315 (95\% CI 1248 to 1382) days, respectively. Figure 1A,B demonstrates no significant difference in mortality between those with or without MCI in the cohort overall $(\log$ rank $\mathrm{P}=0.089)$ and in the SM group $(\log \operatorname{rank} \mathrm{P}=0.740)$. However, in the HBI group (figure 1C), a significant difference in survival was demonstrated with MCI sufferers experiencing worse survival than those without MCI (log-rank $\mathrm{P}=0.036$ ). Multiple logistic regression performed on the cohort overall revealed that increasing age (OR 1.17 (95\% CI 1.10 to 1.24 ), $\mathrm{P}<0.001$ ) and current smoking (OR 4.97 (95\% CI 1.63 to 15.13$), \mathrm{P}=0.005)$ were predictive of all-cause mortality during study follow-up (table 2). The same predictors were identified on analysis in the SM group. In addition to increasing age, participants in the HBI group were over 5.5 times more likely to die if they had MCI (OR 5.57 (95\% CI 1.10 to 28.1), $\mathrm{P}=0.038$ ). No interaction between randomisation group and MCI status was identified for all-cause mortality (data not shown).

Event-free survival (days alive and out-of-hospital)

Figure 2 shows the unadjusted Kaplan-Meier curves for the primary endpoint (time to mortality and unplanned readmission) of participants with and without MCI in the overall cohort (figure 2A) and in the SM (figure 2B) and HBI (figure 2C) groups separately. During follow-up, 206 individuals died or had an unplanned readmission overall (79.2\%) including 145/169 participants (85.8\%) with MCI and 61/91 (67.0\%) without MCI. Mean number of days alive and out-of-hospital in these groups was 411 days (95\% CI 344 to 477 ) and 554 days (95\% CI 446 to 663) days, respectively. Within the SM group, 110 individuals died or had an unplanned readmission (83.3\%) including $77 / 89$ participants $(86.5 \%)$ with MCI and $33 / 43(76.7 \%)$ without MCI. Mean number of days alive and out-of-hospital in these groups was 422 days (95\% CI 334 to 510) and 498 days (95\% CI 372 to 624), respectively. Within the HBI group, 96 individuals died or had an unplanned readmission $(75.0 \%)$ including $68 / 80$ participants $(85.0 \%)$ with MCI and 28/48 (58.3\%) without MCI. Mean number of days alive and out-of-hospital in these groups was 392 days (95\% CI 294 to 490) and 602 days (95\% CI 435 to 769), respectively. No significant difference in time to the primary endpoint was demonstrated in the SM group between those with or without MCI (figure 2B; log rank $\mathrm{P}=0.263$ ). However, in the cohort overall and in the HBI group (figure 2A,C), a significant difference in the number of days alive and out-of-hospital was demonstrated with MCI sufferers experiencing death or unplanned readmission earlier than those without MCI $(\log$-rank $\mathrm{P}=0.012$ and $\mathrm{P}=0.022$, respectively).

On adjusted analyses (table 2) in the overall cohort, age (OR 1.09 (95\% CI 1.05 to 1.13), $\mathrm{P}<0.001$ ), current smoking (OR 2.54 (95\% CI 1.04 to 6.19 ), $\mathrm{P}=0.040$ ) and comorbid type 2 diabetes (OR 2.04 (95\% CI 1.04 to 4.00), 
Table 2 Independent correlates of survival (all-cause mortality and event-free survival via lower quartile of days alive and out-of-hospital) overall and in $\mathrm{HBI}$ and SM groups separately

\begin{tabular}{|c|c|c|c|c|c|c|}
\hline & \multicolumn{3}{|c|}{$\begin{array}{l}\text { All-cause mortality } \\
\text { OR }(95 \% \mathrm{Cl})\end{array}$} & \multicolumn{3}{|c|}{$\begin{array}{l}\text { Lower quartile of days alive and out-of-hospital } \\
\text { OR }(95 \% \mathrm{Cl})\end{array}$} \\
\hline & All & SM & HBI & All & SM & HBI \\
\hline Age (years) & $\begin{array}{l}1.17 \\
(1.10 \text { to } 1.24)^{\star \star}\end{array}$ & $\begin{array}{l}1.20 \\
(1.10 \text { to } 1.31)^{\star \star}\end{array}$ & $\begin{array}{l}1.08 \\
(1.00 \text { to } 1.16)^{*}\end{array}$ & $\begin{array}{l}1.09 \\
(1.05 \text { to } 1.13)^{\star *}\end{array}$ & $\begin{array}{l}1.09 \\
(1.04 \text { to } 1.14)^{\star \star}\end{array}$ & $\begin{array}{l}1.08 \\
(1.02 \text { to } 1.13)^{\star}\end{array}$ \\
\hline Current smoker† & $\begin{array}{l}4.97 \\
(1.63 \text { to } 15.13)^{*}\end{array}$ & $\begin{array}{l}9.63 \\
(2.25 \text { to } 41.21)^{*}\end{array}$ & & $\begin{array}{l}2.54 \\
(1.04 \text { to } 6.19)^{\star}\end{array}$ & $\begin{array}{l}5.07 \\
(1.62 \text { to } 15.91)^{\star}\end{array}$ & - \\
\hline \multicolumn{7}{|l|}{ Comorbidity profile } \\
\hline Type 2 diabetes & $\begin{array}{l}2.23 \\
(0.96 \text { to } 5.19)\end{array}$ & - & - & $\begin{array}{l}2.04 \\
(1.04 \text { to } 4.00)^{*}\end{array}$ & - & $\begin{array}{l}2.97 \\
(1.34 \text { to } 7.77)^{\star}\end{array}$ \\
\hline
\end{tabular}

${ }^{*} \mathrm{P}<0.05$.

${ }^{* *} \mathrm{P}<0.001$.

†Assessed in $\mathrm{n}=258$ patients.

$\mathrm{HBI}$, home-based intervention; SM, standard management.

$\mathrm{P}=0.039)$ were significantly predictive of experiencing the shortest duration of event-free survival (ie, inclusion in the lower quartile of days alive and out-of-hospital). Only age and current smoking were significantly predictive of the same in the SM group (OR 1.09 (95\% CI 1.04 to 1.14 ), $\mathrm{P}<0.001$ and OR 5.07 (95\% CI 1.62 to 15.91 ), $\mathrm{P}=0.005$, respectively). MCI was significantly associated with patients having fewer days alive and out-of-hospital in the HBI group (OR 3.48 (95\% CI 1.06 to 11.4), $\mathrm{P}=0.039)$ in addition to increasing age and comorbid type 2 diabetes (table 2). Online supplementary table 1 demonstrates the results of analyses conducted to understand the interaction between randomisation group and MCI status. Compared with individuals in the HBI group with no MCI, the odds of experiencing the shortest duration of event-free survival for those with MCI in the HBI group were more than tripled and for those with MCI in the SM group, the odds were almost quadrupled.

\section{Hospital readmissions and stay}

All-cause rehospitalisation

Table 3 demonstrates the results of multiple logistic regression analyses identifying independent correlates of all-cause rehospitalisation. In the overall cohort, MCI increased the risk of rehospitalisation due to any cause by over three times during follow-up (OR 3.16 (95\% CI 1.46 to 6.84$), \mathrm{P}=0.003)$. Smoking and type 2 diabetes significantly decreased the risk of all-cause rehospitalisation (OR 0.39 (95\% CI 0.15 to 0.99), $\mathrm{P}=0.048$ and $\mathrm{OR}$ 0.36 (95\% CI 0.15 to 0.83$), \mathrm{P}=0.017$, respectively). In the SM group, risk of all-cause rehospitalisation was significantly increased with increasing age (OR 1.07 (95\% CI
1.02 to 1.34$), \mathrm{P}=0.012$ ) and, again, significantly reduced in smokers and those with type 2 diabetes. The only independent correlate of all-cause rehospitalisation in the HBI group was MCI, which more than tripled the risk of all-cause rehospitalisation (OR 3.30 (95\% CI 1.25 to 8.69$), \mathrm{P}=0.016$ ). Online supplementary table 1 also demonstrates that compared with individuals in the HBI group with no MCI, the odds of experiencing all-cause rehospitalisation for those with MCI in the HBI group were almost quadrupled, and for those with MCI in the SM group, the odds were increased fivefold.

\section{CVD-related rehospitalisation}

Digoxin significantly increased the risk of CVD-related rehospitalisation in the cohort overall and in the SM group. In a similar pattern to all-cause rehospitalisation, the only independent correlate of CVD-related rehospitalisation in the HBI group was MCI (table 3). Those with MCI were over twofold more likely to be hospitalised due to CVD during follow-up (OR 2.35 (95\% CI 1.12 to 4.91), $\mathrm{P}=0.024)$. No interaction between randomisation group and MCI status was identified for CVD-related rehospitalisation (data not shown).

\section{DISCUSSION}

In a typically older and clinically complex $\mathrm{AF}$ patient population, we found that the health outcomes of patients undergoing a nurse-led, AF-specific disease management intervention were negatively impacted by the presence of MCI. Overall, MCI influenced the number of days spent alive and out-of-hospital and all-cause rehospitalisation 
A. Overall study cohort

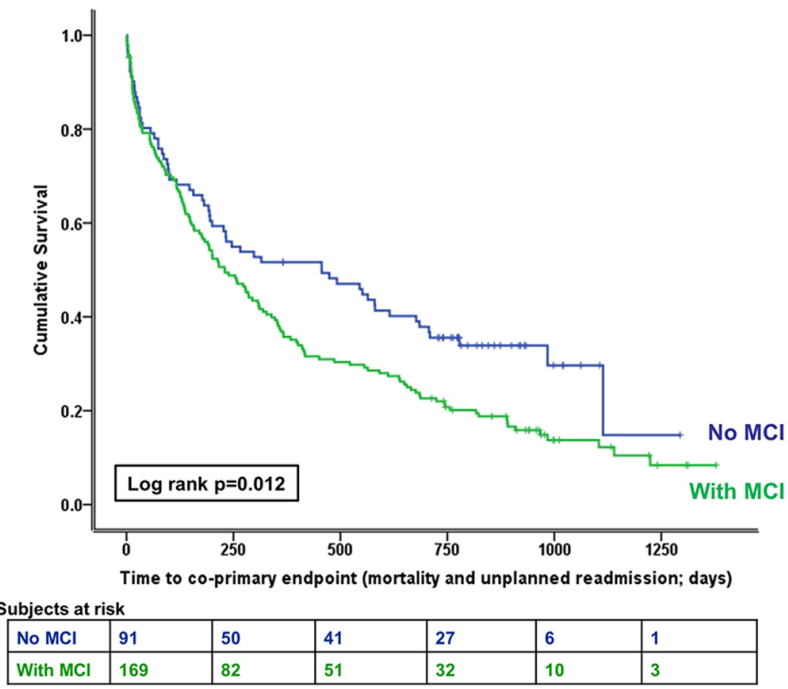

B. Standard management cohort

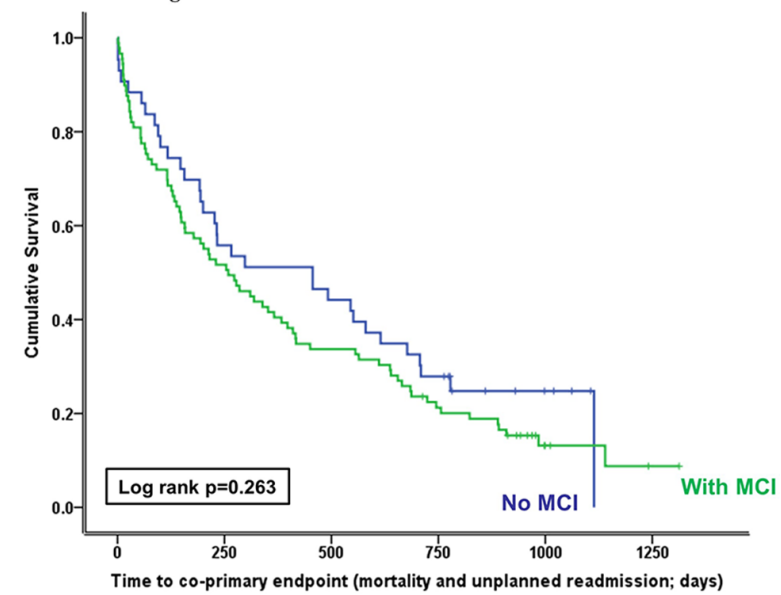

Subjects at risk

\begin{tabular}{|l|l|l|l|l|l|l|}
\hline No MCl & 43 & 24 & 19 & 12 & 4 & 0 \\
\hline With MCl & 89 & 46 & 30 & 18 & 4 & 1 \\
\hline
\end{tabular}

C. Home-based intervention cohort

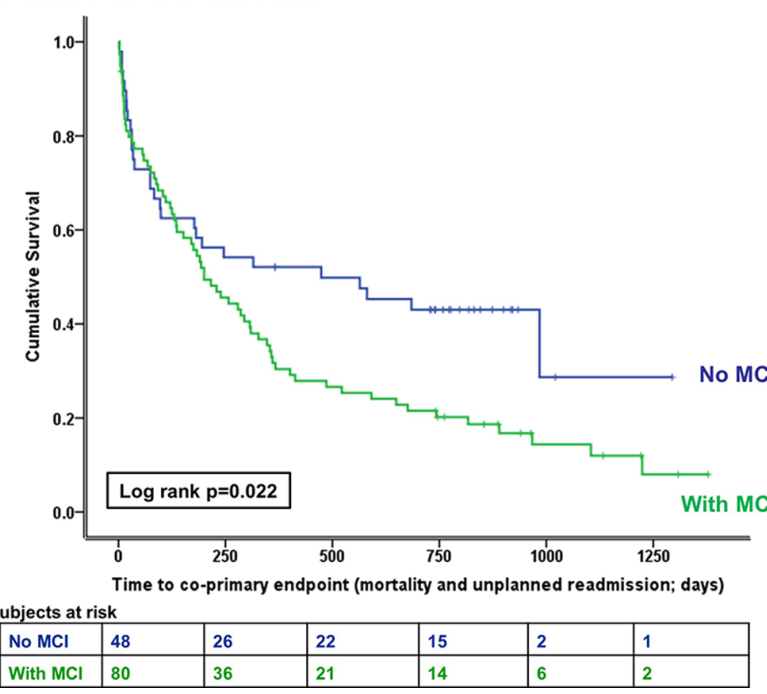

Figure 2 Unadjusted event-free survival (days alive and outof-hospital) of participants with and without $\mathrm{MCl}$ in $(A)$ overall cohort, (B) standard management cohort and (C) homebased intervention cohort. $\mathrm{MCl}$, mild cognitive impairment. of the cohort during follow-up (but not mortality or CVD-related hospitalisation) and did not influence any outcomes for those in the SM group. However, significantly worse outcomes were experienced by those in the HBI group who had MCI. Survival was significantly influenced by MCI; these individuals were at over 5.5 times the risk of dying. This diminished survival was driven, at least in part, by reduced event-free survival; those with MCI in the HBI group were 3.5 times more likely to experience the shortest duration of event-free survival during follow-up. Furthermore, MCI was the only independent correlate of all-cause and CVD-related rehospitalisation in the HBI group. When the interaction between randomisation group and MCI status was analysed, significant differences in event-free survival and all-cause rehospitalisation were demonstrated between those with and without MCI but not between HBI and SM.

To our knowledge, this is the first study to demonstrate a significant disadvantage to health outcomes (survival and rehospitalisations) associated with the implementation of a disease management intervention in those with $\mathrm{AF}$ and MCI. This phenomenon has, however, been identified previously in the context of heart failure. Findings of a study comparing a heart failure-specific disease management intervention with SM demonstrated that MCI was of prognostic importance even when a disease-specific intervention was applied. ${ }^{25}$ Similarly, a study investigating the impact of a self-care training intervention to reduce hospital readmissions in heart failure patients with MCI found that despite increasing patient knowledge, readmission rates were not reduced. ${ }^{26}$ Conversely, in a study conducted by Ketterer and colleagues, a heart failure-specific disease management intervention implemented in patients with comorbid MCI resulted in a 30\% reduction in early readmission compared with a control group. ${ }^{27}$ In this study, those with MCI were provided additional support including conducting a discussion with the patient by all members of their treatment team about the effect of cognitive impairment on adherence to their complex medication regimens, provision of cognitive impairment-specific psychoeducation and the involvement of family members to act as healthcare collaborators. ${ }^{27}$

HBI appears highly effective for patients without MCI but has relatively little benefit in those with MCI. Given that a potent effect of MCI was identified in the HBI group only, it is possible that a disease-specific intervention of this design is too confusing for those who already have difficulty with information processing and executive functioning. It may not be appropriate to apply such a comprehensive (and potentially complex) intervention in this patient group. It is also possible that application of information provided in this format is confronting and may trigger health-related anxiety for those with MCI. The result may be a lack of confidence in self-management, reduced self-care and declining clinical stability. The reasons behind the lack of effectiveness in this patient group is likely to be multifactorial, but 
Table 3 Independent correlates of rehospitalisation (all cause and CVD related) overall and in HBI and SM groups separately $\begin{array}{ll}\text { All-cause rehospitalisation } & \text { CVD-related rehospitalisation } \\ \text { OR }(95 \% \mathrm{Cl}) & \text { OR }(95 \% \mathrm{Cl})\end{array}$

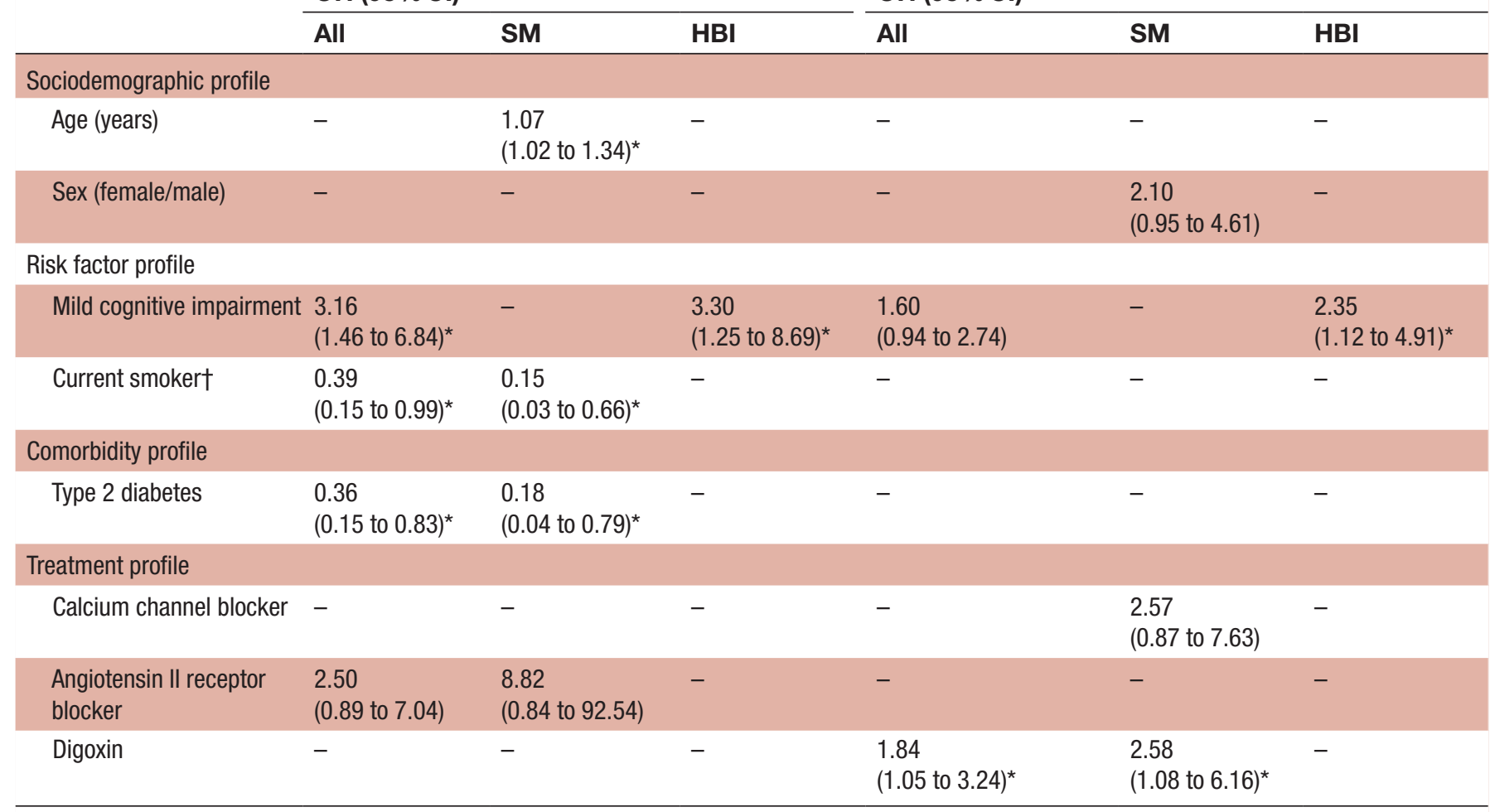

${ }^{*} \mathrm{P}<0.05$.

${ }^{* *} \mathrm{P}<0.001$.

†Assessed in $\mathrm{n}=258$ patients.

CVD, cardiovascular disease; HBI, home based intervention; SM, standard management.

an intervention that is not tailored towards addressing cognitive dysfunction potentially should not be implemented. For the intervention to be effective in patients with $\mathrm{AF}$ and comorbid MCI, integration with healthcare systems (such as psychology and nursing organisations) and a programme design that explicitly caters for cognitively impaired patients is critical. This may also involve care givers more prominently.

Despite the potential for the findings of this work to be considered as part of a 'generic' phenomenon (given it is also seen in heart failure), the high prevalence of MCI in patients with AF means that these individuals should still be considered at high risk for an 'adverse' reaction to $\mathrm{AF}$ (particularly in older individuals) and management must be tailored in response. Therefore, the findings of this study carry substantial clinical significance and support the need for engaging in patient-centred care and individualised, integrated management for those with AF. Evidence-based care of AF patients that maximises effectiveness, safety and efficiency is the ultimate goal and, therefore, enhanced recognition of cognitive impairment and more proactive patient management is necessary. In addition, screening for MCI should become routine practice for patients with $\mathrm{AF}$ with results used to inform the relevance, application and content of AF-specific disease management.

\section{Limitations}

There exist some study limitations that require comment. First, we employed only one method for MCI detection, although the MoCA is a quick and easy tool that demonstrates enhanced sensitivity to early impairment, unlike other screening tools. ${ }^{28}$ Additionally, the entire SAFETY Trial cohort was not screened for MCI due to issues with vision and not originating from an Englishspeaking background; selection bias may have been introduced as a result causing underestimation or overestimation of MCI in this cohort and findings that are not representative. The number of events was also relatively small in the cohort potentially affecting the accuracy and/or generalisability of results. Generalisability may also be affected due to this study being conducted within the Australian urban context with the inclusion of participants principally of European descent. Furthermore, this was a selected population of patients with $\mathrm{AF}$ who did not have heart failure, a common comorbidity. Age, thromboembolic risk $\left(\mathrm{CHA}_{2} \mathrm{DS}_{2}\right.$-VASc score $)$ and severity of comorbidity (Charlson Comorbidity Index) was significantly different at baseline between those with and without MCI in both study groups, although these factors were adjusted for in all statistical models. Finally, we acknowledge that differences detected in this study 
may have arisen due to multiple testing and type I error. However, this was a hypothesis-driven statistical analysis (rather than exploratory) and is consistent with previous findings in heart failure cohorts. Using a more conservative statistical approach (eg, applying Bonferonni correction) would have reduced study power, which needs to be conserved given the number of events observed and stratification of the cohort into those with and without MCI. Therefore, the statistical analyses conducted were appropriate and consistent with the data.

\section{CONCLUSIONS}

We found that the benefit of a home-based, AF-specific disease management intervention for patients with AF was affected by patient cognitive function. MCI was associated with increased mortality and healthcare usage of patients with chronic forms of AF. Therefore, routine screening and detection is critical to direct patient care and optimise management. Future research is required to: (1) further investigate the influence of MCI particularly on the selfcare and self-management of patients with AF; (2) further elucidate the relationship between MCI and different forms of AF patient management; and (3) assess the effectiveness of an AF-specific disease management intervention that includes adjunctive support for patients with MCI.

Contributors All authors contributed to the study design, data analysis, manuscript writing, reviewing and editing.

Funding The SAFETY Trial was funded by a National Health and Medical Research Council of Australia Program Grant (519823). JB (APP1112829) and SS (APP1041796) are supported by the National Health and Medical Research Council of Australia. JB is also supported by a postdoctoral fellowship (Award Reference 100950) from the National Heart Foundation of Australia, and MJC is supported by a Future Leader Fellowship (Award Reference 100802) from the National Heart Foundation of Australia. Supported in part by the Victorian Government's Operational Infrastructure Support Program.

Competing interests None declared.

Patient consent Obtained.

Ethics approval This study was approved by the human research ethics committee(s) associated with each of the study sites (hospitals)

Provenance and peer review Not commissioned; externally peer reviewed.

Data sharing statement No additional data are available.

Open Access This is an Open Access article distributed in accordance with the Creative Commons Attribution Non Commercial (CC BY-NC 4.0) license, which permits others to distribute, remix, adapt, build upon this work non-commercially, and license their derivative works on different terms, provided the original work is properly cited and the use is non-commercial. See: http://creativecommons.org/ licenses/by-nc/4.0/

(c) Article author(s) (or their employer(s) unless otherwise stated in the text of the article) 2018. All rights reserved. No commercial use is permitted unless otherwise expressly granted.

\section{REFERENCES}

1. Ball J, Carrington MJ, McMurray JJ, et al. Atrial fibrillation: profile and burden of an evolving epidemic in the 21 st century. Int $J$ Cardiol 2013;167:1807-24.

2. Stewart S, Hart CL, Hole DJ, et al. A population-based study of the long-term risks associated with atrial fibrillation: 20-year follow-up of the Renfrew/Paisley study. Am J Med 2002;113:359-64.

3. Thrall G, Lane D, Carroll D, et al. Quality of life in patients with atrial fibrillation: a systematic review. Am J Med 2006;119:448.e1-19.
4. Chen LY, Lopez FL, Gottesman RF, et al. Atrial fibrillation and cognitive decline-the role of subclinical cerebral infarcts: the atherosclerosis risk in communities study. Stroke 2014;45:2568-74.

5. Gaita F, Corsinovi L, Anselmino M, et al. Prevalence of silent cerebral ischemia in paroxysmal and persistent atrial fibrillation and correlation with cognitive function. J Am Coll Cardiol 2013;62:1990-7.

6. Gross AF, Stern TA. The cognitive impact of atrial fibrillation. Prim Care Companion CNS Disord 2013;15.

7. Lei C, Lin S, Tao W, et al. Association between cerebral microbleeds and cognitive function: a systematic review. J Neurol Neurosurg Psychiatry 2013;84:693-7.

8 de la Torre JC. Cardiovascular risk factors promote brain hypoperfusion leading to cognitive decline and dementia. Cardiovasc Psychiatry Neurol 2012;2012:1-15.

9. Ball J, Carrington MJ, Stewart S. Mild cognitive impairment in highrisk patients with chronic atrial fibrillation: a forgotten component of clinical management? Heart 2013;99:542-7.

10. Tiwari S, Løchen ML, Jacobsen BK, et al. Atrial fibrillation is associated with cognitive decline in stroke-free subjects: the Troms $\varnothing$ Study. Eur J Neurol 2017;24:1485-92.

11. Kwok CS, Loke YK, Hale R, et al. Atrial fibrillation and incidence of dementia: a systematic review and meta-analysis. Neurology 2011;76:914-22.

12. Flaker GC, Pogue J, Yusuf S, et al. Cognitive function and anticoagulation control in patients with atrial fibrillation. Circ Cardiovasc Qual Outcomes 2010;3:277-83.

13. Dodson JA, Truong TT, Towle VR, et al. Cognitive impairment in older adults with heart failure: prevalence, documentation, and impact on outcomes. Am J Med 2013;126:120-6.

14. Bostrom JA, Saczynski JS, Hajduk A, et al. Burden of psychosocial and cognitive impairment in patients with atrial fibrillation. Crit Pathw Cardiol 2017;16:71-5.

15. Zhu CW, Sano M, Ferris SH, et al. Health-related resource use and costs in elderly adults with and without mild cognitive impairment. $J$ Am Geriatr Soc 2013;61:396-402.

16. Bassuk SS, Wypij D, Berkman LF. Cognitive impairment and mortality in the community-dwelling elderly. Am J Epidemiol 2000;151:676-88.

17. Lyketsos CG, Lopez O, Jones B, et al. Prevalence of neuropsychiatric symptoms in dementia and mild cognitive impairment: results from the cardiovascular health study. JAMA 2002;288:1475-83.

18. Kirchhof P, Benussi S, Kotecha D, et al. ESC Guidelines for the management of atrial fibrillation developed in collaboration with EACTS. Eur Heart J 2016;37:2893-962.

19. Carrington MJ, Ball J, Horowitz JD, et al. Navigating the fine line between benefit and risk in chronic atrial fibrillation: rationale and design of the Standard versus Atrial Fibrillation spEcific managemenT studY (SAFETY). Int J Cardiol 2013;166:359-65.

20. Stewart S, Ball J, Horowitz JD, et al. Standard versus atrial fibrillation-specific management strategy (SAFETY) to reduce recurrent admission and prolong survival: pragmatic, multicentre, randomised controlled trial. Lancet 2015;385:775-84.

21. Zwarenstein M, Treweek S, Gagnier JJ, et al. Improving the reporting of pragmatic trials: an extension of the CONSORT statement. BMJ 2008;337:a2390.

22. Lip GY, Nieuwlaat R, Pisters R, et al. Refining clinical risk stratification for predicting stroke and thromboembolism in atrial fibrillation using a novel risk factor-based approach: the euro heart survey on atrial fibrillation. Chest 2010;137:263-72.

23. Sundararajan V, Henderson T, Perry C, et al. New ICD-10 version of the Charlson comorbidity index predicted in-hospital mortality. J Clin Epidemiol 2004;57:1288-94.

24. Nasreddine ZS, Phillips NA, Bédirian V, et al. The Montreal Cognitive Assessment, MoCA: a brief screening tool for mild cognitive impairment. J Am Geriatr Soc 2005;53:695-9.

25. McLennan SN, Pearson SA, Cameron J, et al. Prognostic importance of cognitive impairment in chronic heart failure patients: does specialist management make a difference? Eur J Heart Fail 2006;8:494-501.

26. Davis KK, Mintzer M, Dennison Himmelfarb CR, et al. Targeted intervention improves knowledge but not self-care or readmissions in heart failure patients with mild cognitive impairment. Eur $J$ Heart Fail 2012;14:1041-9.

27. Ketterer MW, Peltzer J, Mossallam U, et al. Cognitive impairment and reduced early readmissions in congestive heart failure? Am J Manag Care 2016;4:e1-e6.

28. Petersen RC. Mild Cognitive Impairment. N Engl J Med Overseas Ed 2011;364:2227-34. 